\title{
Variações na apresentação fenotípica da escoliose idiopática do adolescente
}

\author{
Variations in the phenotypic presentation of adolescent \\ idiopathic scoliosis
}

Discordancia fenotípica en gemelos monocigóticos

\author{
David Del Curto' \\ Renato Hiroshi Salvioni Ueta ${ }^{1}$ \\ Marcelo Wajchenberg ${ }^{2}$ \\ Délio Eulálio Martins Filho² \\ Eduardo Barros Puertas ${ }^{3}$
}

\section{RESUMO}

Objetivo: discutir quais elementos, de acordo com a literatura, são responsáveis pela discordância fenotípica em gêmeos monozigóticos. Métodos: foram levantados os dados ambulatoriais de um par de gêmeas monozigóticas, que incluíram: idade no momento do diagnóstico, tipo de curva, ângulo de Cobb da deformidade na consulta inicial, início do tratamento e último acompanhamento, ápice da curva e ângulo de Cobb aferido nas imagens radiográficas em perfil. Resultados: criança I: curva principal lombar à esquerda, com ân-

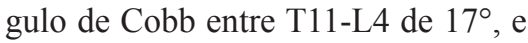
curva torácica direita entre T5-T11 de $14^{\circ}$. Os ápices encontravam-se no disco L1-L2 e na vértebra T8, respectivamente. Um ano depois, se detectou progressão significativa da deformidade, com a curva lombar evoluindo para $24^{\circ}$ (T11-L4) e a curva torácica para $23^{\circ}$ (T5-T11). Criança II: curva toracolombar de pequena magnitude

\section{ABSTRACT}

Objective: to discuss which elements, according to literature, are responsible for phenotypic discordance in monozygotic twins. Methods: the data from a pair of female monozygotic twins were gathered. These data included their age at the time of the diagnosis, type of curve, Cobb angle of the deformity at the time of the initial consultation, dates of the start of treatment and the last follow-up, the apex of the curve and Cobb angle measured from radiological images in lateral view. Results: child I: major lumbar curve to the left, with a Cobb angle of $17^{\circ}$ between T11 and L4, and a thoracic curve to the right, with an angle of $14^{\circ}$ between T5 and T11. The apices were at the L1-L2 disc and at the T8 vertebra, respectively. One year after the first consultation, there had been significant progression of the deformity, with the lumbar curve of $24^{\circ}$ (T11-L4) and the thoracic curve of $23^{\circ}$ (T5-T11). Child II: small
\end{abstract}

\section{RESUMEN}

Objetivo: discutir los elementos que, de acuerdo a la literatura, son responsables por la discordancia fenotípica en gemelos monocigóticos. Métodos: fueron recogidos los datos de un par de pacientes gemelas monocigóticas, que incluyeron la edad al diagnóstico, el tipo de curva, el ángulo de Cobb de la deformidad en la presentación, al inició del tratamiento y a la última consulta de seguimiento, el ápice de la curva y el ángulo de Cobb medido en las imágenes en el perfil. Resultados: niña I - curva lumbar primaria a la izquierda con el ángulo de Cobb entre T11-L4 de $17^{\circ}$ y la curva torácica derecha entre T5-T11 de $14^{\circ}$. Los ápices estaban en el disco L1-L2 y en la vértebra $T 8$, respectivamente. Un año después, se detectó progresión significativa de la deformidad con la curva lumbar progresando a $24^{\circ}$ (T11-L4), y la curva torácica a $23^{\circ}$ (T5-T11). Niña II: curva toracolumbar de pequeña magnitud a la dere-

\footnotetext{
Trabalho realizado na Escola Paulista de Medicina da Universidade Federal de São Paulo - UNIFESP - São Paulo (SP), Brasil; Trabalho original de pôster apresentado no XII Congresso da Sociedade Brasileira de Coluna, realizado no período de 29 de abril a 2 de maio de 2009, em Foz do Iguaçu (PR), Brasil.

'Pós-graduando (Mestrado) do Programa de Pós-graduação em Ortopedia e Traumatologia da Escola Paulista de Medicina da Universidade Federal de São Paulo - UNIFESP - São Paulo (SP), Brasil.

${ }^{2}$ Pós-graduando (Doutorado) do Programa de Pós-graduação em Ortopedia e Traumatologia da Escola Paulista de Medicina da Universidade Federal de São Paulo - UNIFESP - São Paulo (SP), Brasil.

${ }^{3}$ Livre-docente; Professor associado do Departamento de Ortopedia e Traumatologia da Escola Paulista de Medicina da Universidade Federal de São Paulo UNIFESP - São Paulo (SP), Brasil.
} 
à direita, com ângulo de Cobb entre T9 e L3 de $18^{\circ}$. O ápice situava-se na vértebra de T12. Um ano depois, observou-se aumento da curva, com o ângulo de Cobb progredindo para $40^{\circ}$. Conclusão: não obstante a evidência da origem genética para o desenvolvimento da escoliose, admite-se a influência de outros fatores para sua manifestação e progressão. Na literatura, encontram-se algumas explicações para o desenvolvimento da doença, referentes à deficiência de tecidos estruturais encontrada em síndromes e condições específicas, crescimento assimétrico dos membros e tronco, alterações da configuração sagital da coluna vertebral e fatores ligados à natureza, como alimentação.

\section{DESCRITORES: Escoliose/}

etiologia; Escoliose/genética; Doenças em gêmeos thoracolumbar deformity to the right, which was confirmed radiographically with a Cobb angle of $18^{\circ}$ between T9 and L3. The apex was located at the T12 vertebra. One year later, it was observed that the curve had increased, and the Cobb angle had become $40^{\circ}$. Conclusion: notwithstanding the evidence for a genetic origin for the development of scoliosis, it is accepted that other factors influence its manifestation and progression. In literature, the development of this disease has been explained in terms of the structural tissue deficiencies found in specific syndromes and conditions, asymmetrical growth of limbs and trunk, changes in sagittal configuration of the spine and factors relating to the environment, such as nutrition.

KEYWORDS: Scoliosis/etiology; Scoliosis/genetics; Diseases in twins cha, con ángulo de Cobb entre T9 y L3 de $18^{\circ}$. El ápice se encuentra en la vértebra T12. Después de un año, hubo un aumento de la curva, con el ángulo de Cobb progresando hasta $40^{\circ}$. Conclusión: pese a las evidencias del origen genético para el desarrollo de la escoliosis, se admite la influencia de otros factores para su manifestación y progresión. En la literatura hay explicaciones para el desarrollo de la enfermedad que se refieren a la deficiencia de los tejidos estructurales, los cuales se encuentran en sindromes y condiciones específicas, al crecimiento asimétrico de las extremidades y del tronco, a cambios de la configuración sagital de la columna, y a factores relacionados con la naturaleza, como la nutrición.

DESCRIPTORES: Escoliosis/ etiología; Escoliosis/genética; Enfermedades en gemelos

\section{INTRODUÇÃO}

A escoliose idiopática do adolescente (EIA) é um distúrbio musculoesquelético, caracterizado por deformidade tridimensional da coluna vertebral, com uma curvatura lateral associada à rotação dos corpos vertebrais. O diagnóstico é realizado por volta dos dez anos de idade e a maturidade esquelética.

Apesar do grande avanço na compreensão de seu comportamento e do estudo das técnicas de correção, fixação e tecnologia dos implantes, sua etiologia ainda permanece desconhecida.

Alguns trabalhos publicados sugeriram um padrão de herança familiar associado a origem multifatorial ${ }^{1,2}$. Desta forma, teríamos uma doença de etiologia genética com modificações provocadas pelo meio ambiente.

Devido à capacidade de minimizar os efeitos ambientais, estudos com gêmeos foram realizados numa tentativa de estabelecer a participação e a importância da genética na expressão fenotípica da EIA, sendo observada concordância mais significativa entre gêmeos monozigóticos do que em gêmeos dizigóticos ${ }^{3,4}$.

Não obstante a evidência da origem genética para o desenvolvimento da escoliose, admite-se a influência de outros fatores para sua manifestação e progressão ${ }^{5}$. Na literatura, encontram-se algumas explicações para o desenvolvimento da doença, referentes à deficiência de tecidos estruturais encontrada em síndromes e condições específicas, crescimento assimétrico dos membros e tronco, alterações da configuração sagital da coluna vertebral e fatores ligados à natureza, como alimentação.

Apresentamos, neste trabalho, duas pacientes gêmeas monozigóticas concordantes para EIA, porém com diferenças no padrão e na gravidade da curva. Temos como objetivo discutir quais elementos, de acordo com a literatura, são responsáveis pela discordância fenotípica em gêmeos monozigóticos.

\section{MÉTODOS}

Foram levantados os dados de um par de gêmeas monozigóticas, no ambulatório do Grupo de Coluna, que incluíram idade no momento do diagnóstico, tipo de curva, ângulo de Cobb da deformidade na consulta inicial, início do tratamento e último acompanhamento, ápice da curva e ângulo de Cobb aferido nas imagens em perfil.

A classificação das irmãs como gêmeas monozigóticas foi determinada pela avaliação da datiloscopia e similaridade das características físicas. Nenhuma delas possuía qualquer outra anormalidade clínica na coluna ou nas imagens radiográficas.

\section{RESULTADOS}

\section{Paciente 1}

A paciente foi encaminhada para avaliação com especialista após ter sido notada assimetria no tórax por sua professora. Apresentou-se para consulta inicial aos dez 
anos de idade e, no exame físico, observou-se pequena deformidade torácica com giba à direita, confirmada pelo teste de Adams. Não apresentava alterações no exame neurológico.

Nas radiografias da coluna em anteroposterior, apresentou curva principal lombar à esquerda, com ângulo de Cobb entre T11-L4 de $17^{\circ}$, e curva torácica direita entre T5-T11 de $14^{\circ}$. Os ápices encontravam-se no disco L1-L2 e na vértebra T8, respectivamente (Figura 1).

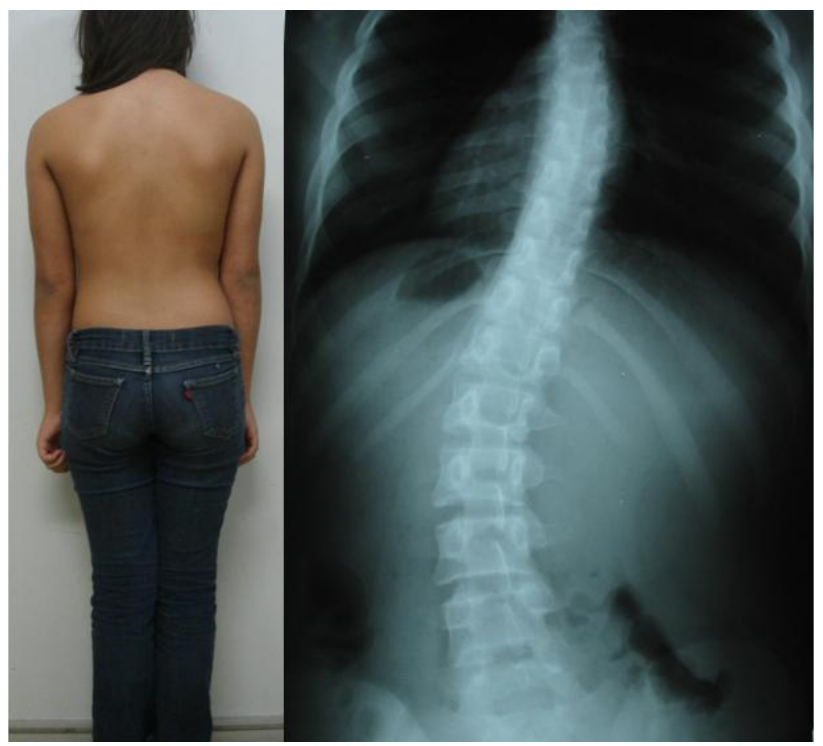

Figura 1

Aspecto físico e radiografia da coluna vertebral, em anteroposterior da paciente 1, mostrando escoliose idiopática com curva principal lombar esquerda.

Um ano após a primeira consulta, foi novamente avaliada e, ao exame radiológico, detectou-se progressão significativa da deformidade, com a curva lombar evoluindo para $24^{\circ}$ (T11-L4) e a curva torácica para $23^{\circ}$ (T5-T11).

A paciente, nesse momento do acompanhamento, tinha estatura de $1,57 \mathrm{~m}$ e $51 \mathrm{~kg}$ de peso e, assim como sua irmã, praticava natação há um mês. Devido à progressão da curva e levando-se em consideração que a curva torácica mantinha o ápice em T8, indicou-se tratamento com colete do tipo TLSO.

\section{Paciente 2}

Após avaliação pela mesma professora, foi encaminhada para ambulatório de especialidade junto com a irmã gêmea. Ao exame físico, apresentava deformidade toracolombar de pequena magnitude à direita, confirmada radiograficamente, com ângulo de Cobb entre T9 e L3 de $18^{\circ}$. O ápice situava-se na vértebra T12 (Figura 2).

Um ano depois, observou-se aumento da curva, com o ângulo de Cobb progredindo para $40^{\circ}$. A estatura e peso dessa paciente eram ligeiramente menores: $1,53 \mathrm{~m}$ e $45 \mathrm{~kg}$, respectivamente. Nesse momento, já se encontrava usando colete tipo TLSO.

Do ponto de vista da maturidade esquelética, ambas encontram-se na escala de Risser 0 . No entanto, apesar de

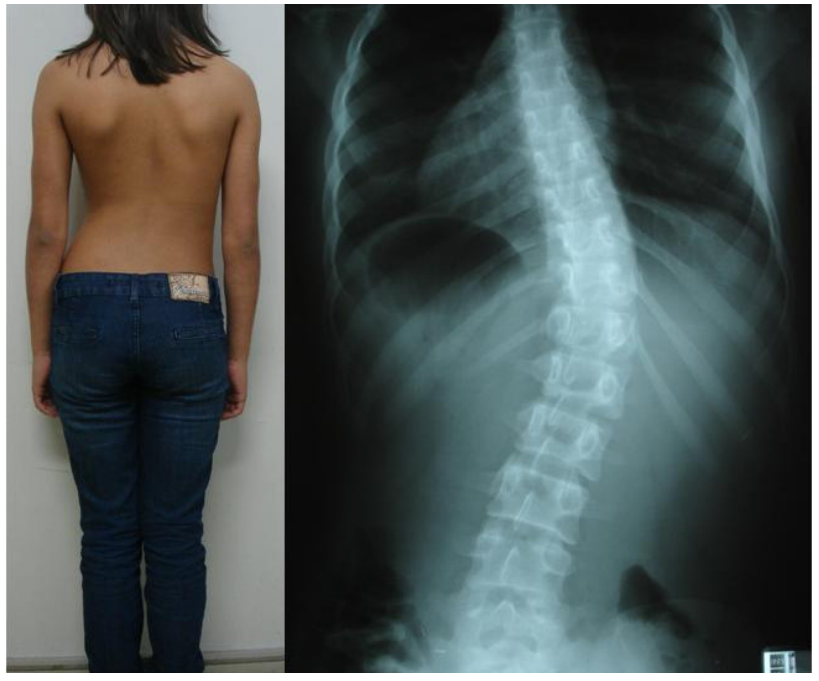

Figura 2

Aspecto físico e radiografia da coluna vertebral, em anteroposterior da paciente 2, mostrando escoliose idiopática com curva toracolombar direita.

terem apresentado suas menarcas entre as duas consultas, a paciente 2 teve atraso de um mês em relação à paciente 1 .

Quanto aos hábitos de vida diária, a criança 2 diferenciava-se da irmã pelo fato de praticar mais esportes de rotina (basquete e vôlei), não-competitivos, e com uma frequência aproximada de três dias durante a semana, em treinamentos de uma hora de duração.

\section{DISCUSSÃO}

Estudos com gêmeos são descritos desde 1875, quando Galton $^{6}$ concluiu que diferenças encontradas entre irmãos monozigóticos eram atribuídas a fatores ambientais. Esses tipos de estudo detêm valor fundamental quando se pretende investigar a etiologia de certas doenças, sabidamente de origem genética, mas com apresentação fenotípica variável.

A escoliose idiopática, em trabalhos realizados visando encontrar sua etiologia, demonstrou-se fortemente influenciada por fatores genéticos, devido a sua característica peculiar de acometer vários membros de determinadas famílias $^{1,2,7}$. Outros autores, como Carr ${ }^{8}$ e Fisher e De George ${ }^{5}$, propuseram que a influência dos aspectos ambientais era capaz de modificar as características e a gravidade da deformidade em gêmeos monozigóticos. Todavia, essas diferenças comprovaram-se maiores quando confrontadas nos pares dizigóticos.

Considerando-se que concordância significa presença de escoliose em ambos os irmãos e que, para estabelecer o diagnóstico é necessário uma curva igual ou maior que $10^{\circ}$, Kesling e Reinker ${ }^{3}$, em sua revisão sistemática, constataram um índice de concordância de $73 \%$ em 37 pares de gêmeos monozigóticos e de $36 \%$ em 31 pares dizigóticos. Já Inoue et al. ${ }^{4}$ apresentaram uma proporção de $92 \%$ para gêmeos monozigóticos e de $62 \%$ para dizigóticos, além de demonstrar que existem diferenças significativas na gravidade das curvas entre pares mono e dizigóticos. Desta forma, encontraríamos maior semelhança na severidade 
das curvas entre pares de gêmeos monozigóticos do que em dizigóticos.

Em levantamento realizado por Andersen et al. ${ }^{9}$, pelo qual foram identificados pares de gêmeos com EIA, por meio de questionários respondidos à distância, a concordância foi significativamente menor que nos estudos anteriores, logrando um índice de $25 \%$ entre monozigóticos e de zero para dizigóticos. Os autores desse estudo atribuíram essa diferença significativa ao viés de determinação e averiguação incompleta nos trabalhos anteriores, que consistiam em estudos do tipo coorte ou relatos de casos e, por isso, tendiam a superestimar pares monozigóticos concordantes e com doença mais grave.

Uma nova corrente dentro das pesquisas em genética tem apontado os fatores epigenéticos como responsáveis pelas diferenças fenotípicas encontradas em gêmeos monozigóticos, de forma que não somente a expressão individual dos genes é modificada, mas funções cruciais são exercidas, como a determinação da configuração global do organismo e manutenção do padrão de desenvolvimento $^{10}$. Os fatores epigenéticos não estão relacionados com a sequência primária do DNA, existindo três mecanismos principais de alterações relacionadas a este termo: metilação do DNA, modificação das histonas e ação de RNAs não-codificadores. Os padrões de metilação de DNA são os mais estudados e melhor entendidos dentre esses mecanismos, embora modificações de histonas também sejam bastante discutidas. Neste sentido, os fatores ambientais teriam um impacto secundário sobre as diferenças das características de indivíduos geneticamente idênticos ${ }^{11}$. Fraga et $\mathrm{a}^{12}$. demonstraram que os padrões de modificação epigenética divergem, na medida em que os pares de gêmeos monozigóticos envelhecem. Desta forma, ocorreria um acúmulo de defeitos de transmissão da informação epigenética, por meio de divisões celulares sucessivas, de acordo com o envelhecimento cronológico do indivíduo.

Em nosso estudo, encontramos um par de gêmeas monozigóticas e concordantes para EIA. Suas curvas divergiram quanto ao tipo, direção e gravidade, o que denotaria influência do meio ambiente ou ação dos fatores epigenéticos. Uma das pacientes apresentou a menarca com um mês de atraso em relação à irmã, e isso poderia estar relacionado com a progressão mais acentuada da sua deformidade (paciente 2). Apesar disso, e ao contrário do que era esperado, apresentava estatura e peso menores (diferença de $4 \mathrm{~cm}$ e $6 \mathrm{~kg}$, respectivamente) no dia da última consulta.

Essa mesma paciente tinha o hábito de praticar mais esportes que a irmã, numa frequência de três vezes por semana. Estudos na literatura já foram publicados demonstrando um aumento na incidência de escoliose em atletas de alto rendimento, a exemplo do trabalho apresentado por Tanchev et al. ${ }^{13}$, pelo qual $12 \%$ das praticantes de ginástica rítmica tiveram o diagnóstico confirmado por radiografia (um aumento de dez vezes em relação ao esperado na população geral). O mesmo autor sugere uma hipótese etiológica que incluiria a "tríade perigosa" (frouxidão ligamentar generalizada, atraso na maturidade esquelética e sobrecarga assimétrica sobre a coluna) e que seria responsável pela deformidade em alguns adolescentes não praticantes de qualquer modalidade esportiva. Por outro lado, Potoupnis et al. ${ }^{14}$ apresentaram um par de irmãs gêmeas monozigóticas e competidoras de nado sincronizado, com duração e tempo de treinamento equivalente, porém discordantes para EIA. Em seu trabalho, questionaram a relação causa-efeito entre atividade física e escoliose, reafirmando seu padrão multifatorial, e reconhecendo a necessidade de mais estudos para estabelecer qualquer interrelação entre essas entidades.

Entre as limitações de um desenho de estudo tipo coorte retrospectivo ou relato de caso, destaca-se a tendência em superestimar pares de gêmeos monozigóticos, concordantes e com deformidades mais graves, configurando-se, desta forma, um viés de detecção9. Visto que, no estágio atual das pesquisas ainda não se encontrou uma resposta definitiva para a etiologia da EIA - nem de que forma fatores conhecidos, e outros por conhecer, influenciam seu comportamento - estudos com gêmeos e o seu acompanhamento por períodos prolongados ainda são necessários para compreender por que há diferenças no padrão das curvas, na idade de diagnóstico e na gravidade da deformidade ${ }^{15}$. Ainda assim, persiste a expectativa e o reconhecimento de que os avanços no campo da Genética e da Biologia Molecular contribuirão de modo essencial para o progresso na compreensão do comportamento e elucidação da etiologia da Escoliose Idiopática.

\section{REFERÊNCIAS}

1. Wynne-Davies R. Familial (idiopathic) scoliosis. A family survey. J Bone Joint Surg Br. 1968;50(1): 24-30.

2. Riseborough EJ, Wynne-Davies R. A genetic survey of idiopathic scoliosis in Boston, Massachusetts. J Bone Joint Surg Am. 1973;55(5): 974-82.
3. Kesling KL, Reinker KA. Scoliosis in twins. A meta-analysis of the literature and report of six cases. Spine (Phila $\mathrm{Pa}$ 1976). 1997;22(17):2009-14.

4. Inoue M, Minami S, Kitahara $H$, Otsuka Y, Nakata Y, Takaso M, et al. Idiopathic scoliosis in twins studied by DNA fingerprinting: the incidence and type of scoliosis. J Bone Joint Surg Br. 1998;80(2):212-7.
5. Fisher RL, De George FV. A twin study of idiopathic scoliosis. Clin Orthop. 1967;55:117-26.

6. Galton F. The history of twins as a criterion of the relative posers of nature and nurture. Pop Sci Monthly. $1875 ; 8: 345$. 
7. van Rhijn LW, Jansen EJ, Plasmans $\mathrm{CM}$, Veraart BE. Changing curve pattern in infantile idiopathic scoliosis: family report with a follow-up of 15 years. Spine (Phila Pa 1976). 2001;26(16):E373-6.

8. Carr AJ. Adolescent idiopathic scoliosis in identical twins. J Bone Joint Surg Br. 1990;72(6):1077.

9. Andersen MO, Thomsen K, Kyvik KO. Adolescent idiopathic scoliosis in twins: a population-based survey. Spine (Phila Pa 1976). 2007;32(8):927-30.

10.Cavalli G. Chromatin and epigenetics in development: blending cellular memory with cell fate plasticity. Development. 2006;133(11):2089-94.
11. Wong AH, Gottesman II, Petronis A. Phenotypic differences in genetically identical organisms: the epigenetic perspective. Hum Mol Genet. 2005;14 Spec No 1:R11-8.

12.Fraga MF, Ballestar E, Paz MF, Ropero S, Setien F, Ballestar ML, et al. Epigenetic differences arise during the lifetime of monozygotic twins. Proc Natl Acad Sci U S A. 2005;102(30):10604-9.

13. Tanchev PI, Dzherov AD, Parushev AD, Dikov DM, Todorov MB. Scoliosis in rhythmic gymnasts. Spine (Phila Pa 1976). 2000;25(11):1367-72.
14.Potoupnis ME, Kenanidis E, Papavasiliou KA, Kapetanos GA. The role of exercising in a pair of female monozygotic (high-class athletes) twins discordant for adolescent idiopathic scoliosis. Spine (Phila $\mathrm{Pa}$ 1976). 2008;33(17):E607-10.

15.Hermus JP, van Rhijn LW, van Ooij A. Non-genetic expression of adolescent idiopathic scoliosis: a case report and review of the literature. Eur Spine J. 2007;16 Suppl 3:338-41.

\section{Correspondência}

David Del Curto

Rua Borges Lagoa, 783, 5ªndar - Vila Clementino

CEP: 04038-031 - São Paulo (SP), Brasil

Tel.:/Fax: (1 1) 5571-6621

E-mail: ddelcurto@hotmail.com 